UWThPh-2003-23

\title{
Maximal atmospheric neutrino mixing and the small ratio of muon to tau mass
}

\author{
Walter Grimus* \\ Institut für Theoretische Physik, Universität Wien \\ Boltzmanngasse 5, A-1090 Wien, Austria \\ Luís Lavoura ${ }^{\S}$ \\ Universidade Técnica de Lisboa \\ Centro de Física das Interacções Fundamentais \\ Instituto Superior Técnico, P-1049-001 Lisboa, Portugal
}

27 October 2003

\begin{abstract}
We discuss the problem of the small ratio of muon mass to tau mass in a class of seesaw models where maximal atmospheric neutrino mixing is enforced through a $\mu-\tau$ interchange symmetry. We introduce into those models an additional symmetry $K$ such that $m_{\mu}=0$ in the case of exact $K$ invariance. The symmetry $K$ may be softly broken in the Higgs potential, and one thus achieves $m_{\mu} \ll m_{\tau}$ in a technically natural way. We speculate on a wider applicability of this mechanism.
\end{abstract}

\footnotetext{
*E-mail: walter.grimus@univie.ac.at

${ }^{\S}$ E-mail: balio@cfif.ist.utl.pt
} 


\section{Introduction}

Lepton mixing seems to be an established fact now - for reviews see, for instance, Ref. [1]. The solar neutrino mixing has turned out to be large but non-maximal, whereas it is likely that the atmospheric mixing angle is maximal, i.e. $\pi / 4$ or close to that value.

On the theoretical side, a popular way of generating small neutrino masses is the seesaw mechanism [2]. It has three sources of lepton mixing: the charged-lepton mass matrix $M_{\ell}$, the Dirac mass matrix $M_{D}$ linking the left-handed neutrinos $\nu_{L}$ to the righthanded neutrinos $\nu_{R}$, and the Majorana mass matrix $M_{R}$ of the right-handed neutrinos; the mass Lagrangian is

$$
\mathcal{L}_{\text {mass }}=-\bar{\ell}_{R} M_{\ell} \ell_{L}-\bar{\nu}_{R} M_{D} \nu_{L}-\frac{1}{2} \bar{\nu}_{R} M_{R} C \bar{\nu}_{R}^{T}+\text { H.c. }
$$

The mass matrix of the light neutrinos is then given by

$$
\mathcal{M}_{\nu}=-M_{D}^{T} M_{R}^{-1} M_{D}
$$

Some models have been proposed in the literature [3, 4, 5, 6] which account for maximal atmospheric neutrino mixing by invoking non-abelian symmetries such that $M_{\ell}$ and $M_{D}$ are simultaneously diagonal. In this case the mass matrix $M_{R}$ is the sole source of neutrino mixing. An interesting common feature of the models of Refs. [4, 15, 6] is that they need a minimum of three Higgs doublets in order to have enough freedom to accommodate all lepton masses.

In this paper we investigate the " $\mathbb{Z}_{2}$ model" of Ref. [4], the " $D_{4}$ model" of Ref. [5], and the "CP model" of Ref. [6]. The models of Refs. [4, [5] have non-abelian horizontalsymmetry groups. The model of Ref. [6] has a non-standard $C P$ symmetry [7] -for the general idea of non-standard $C P$ transformations see Ref. [8] -instead of a certain $\mathbb{Z}_{2}$ contained in the symmetry groups of Refs. 4, 5. The symmetries defining the models of Refs. 4, 5, 6] will be described in the respective sections of this paper; there, their scalar sectors are also discussed.

In all those models the masses of the charged leptons are free and must be adjusted by finetuning. The finetuning problem of adjusting the small ratio of muon mass $\left(m_{\mu}\right)$ to tau mass $\left(m_{\tau}\right)$ is more complex than in the Standard Model, as we shall see below. In Ref. [5] we have proposed a solution to this problem by introducing an additional symmetry operation $K$-but no additional fields - which restricts the coupling constants of the model in such a way that $m_{\mu}=0$ with exact $K$ invariance. ${ }^{1}$ Through soft $K$ breaking a non-zero ratio $m_{\mu} / m_{\tau}$ is then achieved, and this solves the finetuning problem in a technically natural way. The problem of reconciling maximal atmospheric neutrino mixing with a small ratio $m_{\mu} / m_{\tau}$ was also addressed in Ref. 9] in a class of models different from the ones discussed here.

The purpose of this paper is twofold: firstly, we present the detailed proof of the existence of this mechanism and work out the principles on which it is based; secondly,

\footnotetext{
${ }^{1}$ In the paper of Ref. [5] we have used $T$ instead of $K$ for denoting the extra symmetry which leads to $m_{\mu}=0$. Since $T$ is commonly used in physics for the time-reversal symmetry, in this paper and in Ref. [6] we have switched the notation to $K$.
} 
we show that it may operate not only in the model of Ref. [5] but also in those of Refs. [4, 6]. It will become evident to which class of models the symmetry $K$ can be applied.

In Section 2 we describe the multiplets and Yukawa Lagrangians of the models of Refs. 4, 5, 6, we point out the finetuning problem for $m_{\mu} \ll m_{\tau}$, and we introduce the symmetry $K$, which reduces the finetuning to a problem of achieving nearly equal vacuum expectation values (VEVs) for two Higgs doublets. The latter problem is addressed by investigating the effect of $K$ on the Higgs potentials of the $\mathbb{Z}_{2}, D_{4}$, and $C P$ models in Sections [3, 4, and 5, respectively. Section [6 contains a summary.

\section{The Yukawa Lagrangians and $K$}

In Refs. 4, 5] there are the following Yukawa couplings in the lepton sector:

$$
\begin{aligned}
\mathcal{L}_{\mathrm{Y}}= & -y_{1} \bar{D}_{e} \nu_{e R} \tilde{\phi}_{1}-y_{2}\left(\bar{D}_{\mu} \nu_{\mu R}+\bar{D}_{\tau} \nu_{\tau R}\right) \tilde{\phi}_{1} \\
& -y_{3} \bar{D}_{e} e_{R} \phi_{1}-y_{4}\left(\bar{D}_{\mu} \mu_{R}+\bar{D}_{\tau} \tau_{R}\right) \phi_{2}-y_{5}\left(\bar{D}_{\mu} \mu_{R}-\bar{D}_{\tau} \tau_{R}\right) \phi_{3}+\text { H.c. }
\end{aligned}
$$

The constants $y_{i}(i=1, \ldots, 5)$ are in general complex. Denoting the VEV of $\phi_{j}^{0}(j=$ $1,2,3)$ by $v_{j} / \sqrt{2}$, the muon and tau masses are given by

$$
m_{\mu}=\frac{1}{\sqrt{2}}\left|y_{4} v_{2}+y_{5} v_{3}\right|, \quad m_{\tau}=\frac{1}{\sqrt{2}}\left|y_{4} v_{2}-y_{5} v_{3}\right|
$$

respectively.

A variant of the Lagrangian of Eq. (3), namely

$$
\begin{aligned}
\mathcal{L}_{\mathrm{Y}}^{\prime}= & -y_{1} \bar{D}_{e} \nu_{e R} \tilde{\phi}_{1}-\left(y_{2} \bar{D}_{\mu} \nu_{\mu R}+y_{2}^{*} \bar{D}_{\tau} \nu_{\tau R}\right) \tilde{\phi}_{1} \\
& -y_{3} \bar{D}_{e} e_{R} \phi_{1}-\left(y_{4} \bar{D}_{\mu} \mu_{R}+y_{4}^{*} \bar{D}_{\tau} \tau_{R}\right) \phi_{2}-\left(y_{5} \bar{D}_{\mu} \mu_{R}-y_{5}^{*} \bar{D}_{\tau} \tau_{R}\right) \phi_{3}+\text { H.c. }
\end{aligned}
$$

is found in the model of Ref. [6]. Here, $y_{1}$ and $y_{3}$ are real, but the remaining $y_{i}$ are in general complex; the muon and tau masses are given by

$$
m_{\mu}=\frac{1}{\sqrt{2}}\left|y_{4} v_{2}+y_{5} v_{3}\right|, \quad m_{\tau}=\frac{1}{\sqrt{2}}\left|y_{4}^{*} v_{2}-y_{5}^{*} v_{3}\right|,
$$

respectively.

In all three models under discussion there is an "auxiliary" symmetry of the $\mathbb{Z}_{2}$ type:

$$
\mathbb{Z}_{2}^{(\text {aux })}: \nu_{e R}, \nu_{\mu R}, \nu_{\tau R}, \phi_{1}, e_{R} \text { change sign. }
$$

It is spontaneously broken by $v_{1}$ and it restricts the couplings of the Higgs doublets $\phi_{j}$ as seen in the Yukawa Lagrangians of Eqs. (3) and (5).

One must use finetuning for obtaining $m_{\mu} \ll m_{\tau}$. In the case of the Lagrangians of Eqs. (3) and (15) one does not simply have to choose one Yukawa coupling to be small, like in the Standard Model, rather one has to choose two products of unrelated quantitiesone Yukawa coupling and one VEV - such that in $m_{\mu}$ those two products nearly cancel. 
In order to soften the amount of finetuning we have proposed in Ref. [5] to add to the model of that paper the symmetry

$$
K: \quad \mu_{R} \rightarrow-\mu_{R}, \quad \phi_{2} \leftrightarrow \phi_{3}
$$

That symmetry leads to

$$
y_{4}=-y_{5}
$$

in both the Lagrangians of Eqs. (3) and (15). In this way the finetuning is confined to the VEVs:

$$
\frac{m_{\mu}}{m_{\tau}}=\left|\frac{v_{2}-v_{3}}{v_{2}+v_{3}}\right|
$$

The symmetry $K$ also has effects upon the scalar potential $V$, which we now turn to investigate. In this task, there are some differences among the scalar sectors and among the symmetries of the models of Refs. [4, 5, 6] which must be taken into consideration.

\section{The $\mathbb{Z}_{2}$ model}

The scalar sector of the model of Ref. 4] consists solely of the three Higgs doublets $\phi_{j}$. Its symmetries are the following:

- the three groups $U(1)_{L_{\alpha}}(\alpha=e, \mu, \tau)$ associated with the lepton numbers $L_{\alpha}$, which are softly broken by the Majorana mass terms of the right-handed neutrino singlets, i.e. by the matrix $M_{R}$;

- a $\mathbb{Z}_{2}$-type symmetry given by

$$
\mathbb{Z}_{2}^{(\operatorname{tr})}: D_{\mu} \leftrightarrow D_{\tau}, \mu_{R} \leftrightarrow \tau_{R}, \nu_{\mu R} \leftrightarrow \nu_{\tau R}, \phi_{3} \rightarrow-\phi_{3}
$$

which transposes the second and third families and is spontaneously broken by $v_{3}$;

- the symmetry $\mathbb{Z}_{2}^{(\text {aux })}$ of Eq. (71).

Because of the two $\mathbb{Z}_{2}$-type symmetries the Higgs potential $V$ of this model is invariant under the three independent sign changes

$$
\phi_{j} \rightarrow-\phi_{j}, \quad \phi_{j^{\prime}} \rightarrow \phi_{j^{\prime}} \quad\left(j^{\prime} \neq j\right)
$$

where $j, j^{\prime}=1,2,3$. Therefore, in every term of $V$ each Higgs doublet can occur only either two or four times.

We define the Higgs potential $V_{\phi}$ as the polynomial of order 4 in the $\phi_{j}$ obeying the symmetries of Eqs. (8) and (12). Soft breaking of the symmetry $K$ of Eq. (8) is achieved by adding to $V_{\phi}$ the unique term

$$
V_{\mathrm{soft}}=\mu_{\mathrm{soft}}\left(\phi_{2}^{\dagger} \phi_{2}-\phi_{3}^{\dagger} \phi_{3}\right) .
$$

We want to show the following [5]: 
(a) Under certain conditions on the coupling constants, the minimum of $V_{\phi}$ fulfills

$$
v_{2}=v_{3} .
$$

Equation (10) then gives $m_{\mu}=0$.

(b) The full potential $V=V_{\phi}+V_{\text {soft }}$ leads to a non-vanishing $m_{\mu}$. For small $\mu_{\text {soft }}$ the ratio $m_{\mu} / m_{\tau}$ is small in a technically natural way.

Point (a): $V_{\phi}$ is given by

$$
\begin{aligned}
V_{\phi}= & -\mu_{1} \phi_{1}^{\dagger} \phi_{1}-\mu_{2}\left(\phi_{2}^{\dagger} \phi_{2}+\phi_{3}^{\dagger} \phi_{3}\right) \\
& +\lambda_{1}\left(\phi_{1}^{\dagger} \phi_{1}\right)^{2}+\lambda_{2}\left[\left(\phi_{2}^{\dagger} \phi_{2}\right)^{2}+\left(\phi_{3}^{\dagger} \phi_{3}\right)^{2}\right] \\
& +\lambda_{3}\left(\phi_{1}^{\dagger} \phi_{1}\right)\left(\phi_{2}^{\dagger} \phi_{2}+\phi_{3}^{\dagger} \phi_{3}\right)+\lambda_{4}\left(\phi_{2}^{\dagger} \phi_{2}\right)\left(\phi_{3}^{\dagger} \phi_{3}\right) \\
& +\lambda_{5}\left[\left(\phi_{1}^{\dagger} \phi_{2}\right)\left(\phi_{2}^{\dagger} \phi_{1}\right)+\left(\phi_{1}^{\dagger} \phi_{3}\right)\left(\phi_{3}^{\dagger} \phi_{1}\right)\right]+\lambda_{6}\left(\phi_{2}^{\dagger} \phi_{3}\right)\left(\phi_{3}^{\dagger} \phi_{2}\right) \\
& +\lambda_{7}\left[\left(\phi_{2}^{\dagger} \phi_{3}\right)^{2}+\left(\phi_{3}^{\dagger} \phi_{2}\right)^{2}\right]+\lambda_{8}\left[\left(\phi_{1}^{\dagger} \phi_{2}\right)^{2}+\left(\phi_{1}^{\dagger} \phi_{3}\right)^{2}\right]+\lambda_{8}^{*}\left[\left(\phi_{2}^{\dagger} \phi_{1}\right)^{2}+\left(\phi_{3}^{\dagger} \phi_{1}\right)^{2}\right] .
\end{aligned}
$$

All the coupling constants in $V_{\phi}$, except for $\lambda_{8}$, are real. We replace the Higgs doublets in $V_{\phi}$ by their VEVs, parameterized as

$$
\frac{v_{1}}{\sqrt{2}}=u_{1}, \quad \frac{v_{2}}{\sqrt{2}}=u e^{i \alpha} \cos \sigma, \quad \frac{v_{3}}{\sqrt{2}}=u e^{i \beta} \sin \sigma,
$$

where, without loss of generality, $u_{1}$ and $u$ are positive and $\sigma$ belongs to the first quadrant. Note that $\sqrt{2\left(u_{1}^{2}+u^{2}\right)} \simeq 246 \mathrm{GeV}$ represents the electroweak scale. In this way we obtain the function

$$
\begin{aligned}
F_{\phi} \equiv\left\langle 0\left|V_{\phi}\right| 0\right\rangle= & -\mu_{1} u_{1}^{2}-\mu_{2} u^{2}+\lambda_{1} u_{1}^{4}+\lambda_{2} u^{4}+\left(\lambda_{3}+\lambda_{5}\right) u_{1}^{2} u^{2} \\
& +\left[\tilde{\lambda}-4 \lambda_{7} \sin ^{2}(\alpha-\beta)\right] u^{4} \cos ^{2} \sigma \sin ^{2} \sigma \\
& +2\left|\lambda_{8}\right| u_{1}^{2} u^{2}\left[\cos ^{2} \sigma \cos (\epsilon+2 \alpha)+\sin ^{2} \sigma \cos (\epsilon+2 \beta)\right],
\end{aligned}
$$

where $\tilde{\lambda} \equiv-2 \lambda_{2}+\lambda_{4}+\lambda_{6}+2 \lambda_{7}$ and $\epsilon \equiv \arg \lambda_{8}$. In searching for the minimum of $F_{\phi}$ in terms of $\sigma, \alpha$, and $\beta$ we make the following simple observation: if

$$
\tilde{\lambda}<0 \quad \text { and } \quad \lambda_{7}<0
$$

then the minimum of $F_{\phi}$ has

$$
\sigma=\frac{\pi}{4}, \quad \alpha=\beta=\frac{\pi-\epsilon}{2},
$$

i.e.

$$
v_{2}=v_{3}=u e^{i(\pi-\epsilon) / 2} .
$$

With Eq. (20) one obtains $m_{\mu}=0$, which is close to the real situation if one takes into account that $m_{\mu} \ll m_{\tau}$. Equation (18) formulates sufficient conditions for the minimum of $V_{\phi}$ to obey the relation (20). 
Point (b): Now we take into account $V_{\text {soft }}$, which gives

$$
\left\langle 0\left|V_{\text {soft }}\right| 0\right\rangle=\mu_{\text {soft }} u^{2} \cos 2 \sigma .
$$

We must minimize $\left\langle 0\left|V_{\phi}+V_{\text {soft }}\right| 0\right\rangle$. Since the new term in Eq. (21) does not contain the phases $\alpha$ and $\beta$, the minimum is again at $\alpha=\beta=(\pi-\epsilon) / 2$. Then, the second line of Eq. (17) together with the term stemming from $V_{\text {soft }}$ lead to

$$
\cos 2 \sigma=\frac{2 \mu_{\text {soft }}}{\tilde{\lambda} u^{2}}
$$

It is obvious that, if $\left|\mu_{\text {soft }}\right|$ is sufficiently smaller than $u^{2}$, the angle $\sigma$ will still be close to $\pi / 4$. Using Eqs. (10) and (16), we obtain

$$
\frac{m_{\mu}}{m_{\tau}}=\frac{|\cos 2 \sigma|}{1+\sqrt{1-\cos ^{2} 2 \sigma}} \simeq\left|\frac{\mu_{\text {soft }}}{\tilde{\lambda} u^{2}}\right|,
$$

showing explicitly that a small $\mu_{\text {soft }}$ leads to a small $m_{\mu} / m_{\tau}$.

Let us elaborate on the magnitude of $\mu_{\text {soft }}$. The Higgs-potential coupling $\tilde{\lambda}$ could be of order 0.1. Furthermore, let us choose, for instance, $u=172 \mathrm{GeV}$. Then it turns out that $u_{1} \simeq 26 \mathrm{GeV}$, which is much smaller than $u$; this is reasonable since $u_{1}$ is responsible both for the electron mass and for the neutrino masses. From Eq. (23) one then obtains $\left|\mu_{\text {soft }}\right| \sim 176 \mathrm{GeV}^{2}$. Thus, $\left|\mu_{\text {soft }}\right|$ is much smaller than $u^{2}$, yet it may well be of the order of magnitude or even larger than $u_{1}^{2}$. On the other hand, if $u_{1}$ is comparable to $u$, then we shall have $\left|\mu_{\text {soft }}\right|$ much smaller than both $u^{2}$ and $u_{1}^{2}$, but its order of magnitude will not change with respect to the numerical example above.

\section{The $D_{4}$ model}

The model of Ref. [5] is based on the horizontal-symmetry group $D_{4}$. It has the same gauge multiplets of the model of the previous section, plus two real scalar gauge singlets $\chi_{k}(k=1,2)$. The symmetries are the following: the horizontal group $D_{4}$ is generated by the $\mathbb{Z}_{2}^{(\mathrm{tr})}$ of Eq. (11), supplemented by $\chi_{1} \leftrightarrow \chi_{2}$, and by an additional $\mathbb{Z}_{2}$-type symmetry

$$
\mathbb{Z}_{2}^{(\tau)}: D_{\tau}, \tau_{R}, \nu_{\tau R}, \chi_{2} \text { change sign. }
$$

Besides $D_{4}$, there is also the symmetry $\mathbb{Z}_{2}^{(\text {aux })}$ of Eq. (17). Both $D_{4}$ and $\mathbb{Z}_{2}^{(\text {aux })}$ are spontaneously broken. We furthermore impose the symmetry $K$ of Eq. (8).

The full potential has the structure

$$
V=V_{\chi}+V_{\chi \phi}+V_{\phi}+V_{\text {soft }}
$$

where $V_{\chi}$ contains only the fields $\chi_{k}$ whereas the terms in $V_{\chi \phi}$ contain both the $\chi_{k}$ and the $\phi_{j}$. Because $\mathbb{Z}_{2}^{(\text {aux })}$ and $\mathbb{Z}_{2}^{(\mathrm{tr})}$ hold in this case as well, $V_{\phi}$ and $V_{\text {soft }}$ are the same as in the previous section. We will show that-like for $V_{\phi}$ under point (a) in the previous section - there is a minimum of $V_{\chi}+V_{\chi \phi}+V_{\phi}$ for which Eq. (14) holds. Then the procedure of point (b) works in the present model as well. 
The potentials $V_{\chi}$ and $V_{\chi \phi}$ are given by

$$
\begin{aligned}
V_{\chi} & =-\mu\left(\chi_{1}^{2}+\chi_{2}^{2}\right)+\lambda\left(\chi_{1}^{2}+\chi_{2}^{2}\right)^{2}+\lambda^{\prime}\left(\chi_{1}^{2}-\chi_{2}^{2}\right)^{2}, \\
V_{\chi \phi} & =\left(\chi_{1}^{2}+\chi_{2}^{2}\right)\left[\rho_{1} \phi_{1}^{\dagger} \phi_{1}+\rho_{2}\left(\phi_{2}^{\dagger} \phi_{2}+\phi_{3}^{\dagger} \phi_{3}\right)\right]+\eta\left(\chi_{1}^{2}-\chi_{2}^{2}\right)\left(\phi_{2}^{\dagger} \phi_{3}+\phi_{3}^{\dagger} \phi_{2}\right) .
\end{aligned}
$$

In Eqs. (26) and (27) all the coupling constants are real. We parameterize the VEVs of the $\chi_{k}$ in the following way [5]:

$$
\left\langle 0\left|\chi_{1}\right| 0\right\rangle=W \cos \gamma, \quad\left\langle 0\left|\chi_{2}\right| 0\right\rangle=W \sin \gamma
$$

We then obtain

$$
\begin{aligned}
\left\langle 0\left|V_{\chi}+V_{\chi \phi}\right| 0\right\rangle= & -\mu W^{2}+\lambda W^{4}+W^{2}\left(\rho_{1} u_{1}^{2}+\rho_{2} u^{2}\right) \\
& +2 \eta W^{2} u^{2} \cos 2 \gamma \cos \sigma \sin \sigma \cos (\alpha-\beta)+\lambda^{\prime} W^{4} \cos ^{2} 2 \gamma .
\end{aligned}
$$

The minimum of $\gamma$ is found at

$$
\cos 2 \gamma=-\frac{\eta u^{2} \cos \sigma \sin \sigma \cos (\alpha-\beta)}{\lambda^{\prime} W^{2}}
$$

provided $\lambda^{\prime}>0$ and the quantity in the right-hand side of Eq. (30) is smaller than unity in modulus. ${ }^{2}$ Inserting the result of Eq. (30) into Eq. (29) we arrive at

$$
\begin{aligned}
\left\langle 0\left|V_{\chi}+V_{\chi \phi}\right| 0\right\rangle= & -\mu W^{2}+\lambda W^{4}+W^{2}\left(\rho_{1} u_{1}^{2}+\rho_{2} u^{2}\right) \\
& -\frac{\eta^{2}}{\lambda^{\prime}} u^{4} \cos ^{2} \sigma \sin ^{2} \sigma \cos ^{2}(\alpha-\beta) .
\end{aligned}
$$

Adding this expression to $F_{\phi}$ in Eq. (17), we see that this amounts to the replacements $\tilde{\lambda} \rightarrow \tilde{\lambda}-\eta^{2} / \lambda^{\prime}$ and $4 \lambda_{7} \rightarrow 4 \lambda_{7}-\eta^{2} / \lambda^{\prime}$ in Eq. (17). Then the minimum of $V_{\chi}+V_{\chi \phi}+V_{\phi}$ with respect to $\sigma, \alpha$, and $\beta$ is not changed compared to Eq. (19); we have again $v_{2}=v_{3}$ and, therefore, $m_{\mu}=0$. As before, breaking the symmetry $K$ softly through $V_{\text {soft }}$ one achieves a non-zero muon mass. The muon mass is small in a technically natural way by requiring only one parameter, $\mu_{\text {soft }}$, to be small.

\section{The $C P$ model}

The model of Ref. 6] has the same scalar multiplets and nearly the same symmetries as the $\mathbb{Z}_{2}$ model of Section 3 the only difference is that the $\mathbb{Z}_{2}^{(\mathrm{tr})}$ of Eq. (11) is replaced by the non-standard $C P$ symmetry

$$
\begin{aligned}
D_{\alpha} & \rightarrow i S_{\alpha \beta} \gamma^{0} C \bar{D}_{\beta}^{T}, \\
\alpha_{R} & \rightarrow i S_{\alpha \beta} \gamma^{0} C \bar{\beta}_{R}^{T}, \\
\nu_{\alpha R} & \rightarrow i S_{\alpha \beta} \gamma^{0} C \bar{\nu}_{\beta R}^{T}, \quad \text { with } \quad S=\left(\begin{array}{ccc}
1 & 0 & 0 \\
0 & 0 & 1 \\
0 & 1 & 0
\end{array}\right) . \\
\phi_{1,2} & \rightarrow \phi_{1,2}^{*}, \\
\phi_{3} & \rightarrow-\phi_{3}^{*},
\end{aligned}
$$

\footnotetext{
${ }^{2}$ In the model of Ref. [5] we need $W \gg u$ anyway.
} 
Here, $\alpha, \beta=e, \mu, \tau$.

This replacement not only leads to the Yukawa Lagrangian of Eq. (5), slightly different from the one of Eq. (3), but also allows for a more general Higgs potential, due to the complex conjugation in the transformation of the Higgs doublets in Eq. (32). One can show that invariance under the non-standard $C P$ transformation and under the horizontal symmetry $K$ allows, in addition to the terms in $V_{\phi}$ of Eq. (15), two more terms:

$$
\begin{aligned}
V_{9} & =i \lambda_{9}\left[\left(\phi_{1}^{\dagger} \phi_{2}\right)\left(\phi_{1}^{\dagger} \phi_{3}\right)-\left(\phi_{2}^{\dagger} \phi_{1}\right)\left(\phi_{3}^{\dagger} \phi_{1}\right)\right], \\
V_{10} & =i \lambda_{10}\left(\phi_{2}^{\dagger} \phi_{3}-\phi_{3}^{\dagger} \phi_{2}\right)\left(\phi_{2}^{\dagger} \phi_{2}-\phi_{3}^{\dagger} \phi_{3}\right) .
\end{aligned}
$$

The coupling constants $\lambda_{9}$ and $\lambda_{10}$ are real, and now the same holds for $\lambda_{8}$ in Eq. (15), i.e. $\epsilon \equiv \arg \lambda_{8}=0$ or $\pi$. As for the soft breaking of $K$, besides $V_{\text {soft }}$, the $C P$-invariant term

$$
V_{\mathrm{soft}}^{\prime}=i \mu_{\mathrm{soft}}^{\prime}\left(\phi_{2}^{\dagger} \phi_{3}-\phi_{3}^{\dagger} \phi_{2}\right)
$$

is now allowed too. Thus the full potential is $V=V_{\phi}+V_{9}+V_{10}+V_{\text {soft }}+V_{\text {soft }}^{\prime}$. The VEVs of the additional terms in the potential are given by

$$
\begin{aligned}
\left\langle 0\left|V_{9}\right| 0\right\rangle & =-\lambda_{9} u_{1}^{2} u^{2} \sin 2 \sigma \sin (\alpha+\beta), \\
\left\langle 0\left|V_{10}\right| 0\right\rangle & =\lambda_{10} u^{4} \cos 2 \sigma \sin 2 \sigma \sin (\alpha-\beta), \\
\left\langle 0\left|V_{\text {soft }}^{\prime}\right| 0\right\rangle & =\mu_{\text {soft }}^{\prime} u^{2} \sin 2 \sigma \sin (\alpha-\beta) .
\end{aligned}
$$

To find the minimum of the potential, we proceed in analogy to Section 3 . We define a function $\mathcal{F}_{\phi}=F_{\phi}+\left\langle 0\left|V_{9}\right| 0\right\rangle+\left\langle 0\left|V_{10}\right| 0\right\rangle$ and search for its minimum with respect to $\sigma$, $\alpha$, and $\beta$. We find

$$
\sigma=\frac{\pi}{4}, \quad \alpha=\beta=\omega,
$$

which agrees with the minimum of $V_{\phi}$ in Eq. (19), except that the value of $\omega$ is now determined by

$$
2 \lambda_{8} \sin 2 \omega+\lambda_{9} \cos 2 \omega=0 .
$$

Switching on the two soft $K$-breaking terms, the minimum in Eq. (39) gets shifted. Because of the complication with the three additional terms in $V$, we are unable to give an exact solution for the shifted minimum, like we did in Section [3. We therefore resort to a discussion of the shift of the minimum to first order in the small quantities $\mu_{\text {soft }}$ and $\mu_{\text {soft }}^{\prime}$. We define $\delta_{0}, \delta_{+}$, and $\delta_{-}$as characterizing the deviations of $\sigma, \alpha$, and $\beta$ from their values in Eq. (39):

$$
\sigma=\frac{\pi}{4}-\frac{\delta_{0}}{2}, \quad \alpha=\omega+\delta_{+}+\frac{\delta_{-}}{2}, \quad \beta=\omega+\delta_{+}-\frac{\delta_{-}}{2} .
$$

We expand the function $\mathcal{F}_{\phi}$ to second order, and the soft-breaking terms to first order, in these small variables. Dropping the constant terms, we obtain the expansion

$$
\frac{1}{2} \sum_{a, b} \mathcal{F}_{a b} \delta_{a} \delta_{b}+\sum_{a} f_{a} \delta_{a}
$$


where $\mathcal{F} \equiv\left(\mathcal{F}_{a b}\right)$ is the symmetric and positive matrix of the second derivatives of $\mathcal{F}_{\phi}$ at the minimum (39):

$$
\begin{aligned}
\mathcal{F}_{00} & =-\frac{1}{2} \tilde{\lambda} u^{4}+\lambda_{9} u_{1}^{2} u^{2} \sin 2 \omega, \\
\mathcal{F}_{++} & =\left(-8 \lambda_{8} \cos 2 \omega+4 \lambda_{9} \sin 2 \omega\right) u_{1}^{2} u^{2}, \\
\mathcal{F}_{--} & =-2 \lambda_{7} u^{4}-2 \lambda_{8} u_{1}^{2} u^{2} \cos 2 \omega, \\
\mathcal{F}_{0-} & =-2 \lambda_{8} u_{1}^{2} u^{2} \sin 2 \omega+\lambda_{10} u^{4}, \\
\mathcal{F}_{0+} & =0, \\
\mathcal{F}_{+-} & =0 .
\end{aligned}
$$

The second term in Eq. (42) represents the expansion of the soft-breaking terms, with

$$
f_{0}=\mu_{\text {soft }} u^{2}, \quad f_{+}=0, \quad f_{-}=\mu_{\text {soft }}^{\prime} u^{2} .
$$

Let us now define the vectors $\mathbf{f}=\left(f_{0}, f_{+}, f_{-}\right)^{T}$ and $\boldsymbol{\delta}=\left(\delta_{0}, \delta_{+}, \delta_{-}\right)^{T}$. The minimum of $V$ is given, to first order in the $\delta_{a}$ and in the soft-breaking parameters, by

$$
\boldsymbol{\delta}=-\mathcal{F}^{-1} \mathbf{f}
$$

Using Eqs. (45), (43), and (44) it is straightforward to calculate the shifts $\delta_{a}$. From the zeros in $\mathcal{F}$ and $\mathbf{f}$ one immediately concludes that $\delta_{+}=0$, i.e. the quantity $\alpha+\beta$ remains $2 \omega$ to first order in the small ratios $\mu_{\text {soft }} / u_{1}^{2}, \mu_{\text {soft }}^{\prime} / u_{1}^{2}, \mu_{\text {soft }} / u^{2}$, and $\mu_{\text {soft }}^{\prime} / u^{2}$. The ratio of the muon mass to the tau mass is expressed as

$$
\frac{m_{\mu}}{m_{\tau}}=\left|\frac{v_{2}-v_{3}}{v_{2}+v_{3}}\right| \simeq \frac{1}{2}\left|\delta_{0}+i \delta_{-}\right|,
$$

showing that a non-zero muon mass is generated not only by the different absolute values of $v_{2}$ and $v_{3}$, as in Section 3. but also by the different phases of those VEVs.

\section{Summary}

In this paper we have shown that the models of Refs. 4, 5, 6, which achieve maximal atmospheric neutrino mixing through non-abelian symmetries, allow for a technically natural explanation of the smallness of the muon mass as compared to the tau mass.

The models are characterized by diagonal Yukawa couplings, as seen in the Lagrangian of Eq. (3), valid for the models of Refs. [4, 5], and in the Lagrangian of Eq. (15), in the case of Ref. 6]. At face value those Yukawa Lagrangians suggest that $m_{\mu}$ and $m_{\tau}$ should be of the same order of magnitude. In this paper, the key for $m_{\mu} \ll m_{\tau}$ was the extra horizontal symmetry $K$ of Eq. (8) . As a consequence of that symmetry the ratio $m_{\mu} / m_{\tau}$ is given by Eq. (10), which is a function only of the VEVs $v_{2}$ and $v_{3}$. We have shown that the invariance of the Higgs potentials under $K$ and under the additional symmetries of the various models is such that they all admit minima with $v_{2}=v_{3}$, leading to $m_{\mu}=0$. We then break $K$ softly by the term in Eq. (13), which is unique in the cases of the $\mathbb{Z}_{2}$ model of Ref. [4] and of the $D_{4}$ model of Ref. [5]; in the case of the $C P$ model of Ref. [6] there is the additional $K$ soft-breaking term of Eq. (35). In this way, we link the smallness of $m_{\mu} / m_{\tau}$ to the smallness of the soft-breaking terms. 
As for the electron mass, it can be read off from the Yukawa Lagrangians (3) and (5) that the smallness of $m_{e}$ is linked to the smallness of the neutrino masses through the small VEV of $\phi_{1}^{0}$.

We feel that seesaw models where neutrino mixing stems solely from the Majorana mass matrix of the heavy neutrino singlets are quite appealing, since they allow for symmetries that enforce maximal atmospheric neutrino mixing. It is, therefore, possible that our mechanism for $m_{\mu} \ll m_{\tau}$, which is connected with Yukawa Lagrangians of the type in Eqs. (3) and (5) and does not need any new fields, has a wider applicability and is not confined to the models of Refs. [4, 5, 6, discussed in this paper.

Acknowledgement The work of L.L. was supported by the Portuguese Fundação para a Ciência e a Tecnologia under the contract CFIF-Plurianual. 


\section{References}

[1] W. Grimus, Neutrino physics - Theory, lectures given at the 41. Internationale Universitätswochen für Theoretische Physik, Flavour Physics, Schladming, Styria, Austria, 22-28 February 2003 hep-ph/0307149;

J.W.F. Valle, Neutrino masses twenty-five years later, invited talk presented at MRST'03, Syracuse, New York, May 2003 hep-ph/0307192;

V. Barger, D. Marfatia, and K. Whisnant, Progress in the physics of massive neutrinos, hep-ph/0308123.

[2] M. Gell-Mann, P. Ramond, and R. Slansky, Complex spinors and unified theories, in Supergravity, Proceedings of the Workshop, Stony Brook, New York, 1979, eds. P. van Nieuwenhuizen and D.Z. Freedman (North Holland, Amsterdam, 1979);

T. Yanagida, Horizontal gauge symmetry and masses of neutrinos, in Proceedings of the Workshop on Unified Theories and Baryon Number in the Universe, Tsukuba, Japan, 1979, eds. O. Sawada and A. Sugamoto (KEK report no. 79-18, Tsukuba, 1979);

R.N. Mohapatra and G. Senjanović, Neutrino mass and spontaneous parity violation, Phys. Rev. Lett. 44 (1980) 912.

[3] K.S. Babu, E. Ma, and J.W.F. Valle, Underlying $A_{4}$ symmetry for the neutrino mass matrix and the quark mixing matrix, Phys. Lett. B 552 (2003) 207 hep-ph/0206292; see also E. Ma, Plato's fire and the neutrino mass matrix, Mod. Phys. Lett. A 17 (2002) 2361 hep-ph/0211393.

[4] W. Grimus and L. Lavoura, Softly broken lepton numbers and maximal neutrino mixing, J. High Energy Phys. 07 (2001) 045 hep-ph/0105212;

W. Grimus and L. Lavoura, Softly broken lepton numbers: an approach to maximal neutrino mixing, Acta Phys. Polon. B 32 (2001) 3719 hep-ph/0110041.

[5] W. Grimus and L. Lavoura, A discrete symmetry group for maximal atmospheric neutrino mixing, Phys. Lett. B 572 (2003) 189 hep-ph/0305046 v2].

[6] W. Grimus and L. Lavoura, A non-standard CP transformation leading to maximal atmospheric neutrino mixing, to be published in Phys. Lett. B hep-ph/0305309 v3].

[7] P.F. Harrison and W.G. Scott, $\mu-\tau$ reflection symmetry in lepton mixing and neutrino oscillations, Phys. Lett. B 547 (2002) 219 hep-ph/0210197.

[8] G. Ecker, W. Grimus, and W. Konetschny, Quark mass matrices in left-right symmetric gauge theories, Nucl. Phys. B 191 (1981) 465;

G. Ecker, W. Grimus, and H. Neufeld, Spontaneous CP violation in left-right symmetric gauge theories, Nucl. Phys. B 247 (1984) 70;

W. Grimus and M.N. Rebelo, Automorphisms in gauge theories and the definition of $C P$ and P, Phys. Rept. 281 (1997) 239 hep-ph/9506272.

[9] T. Ohlsson and G. Seidl, A flavor symmetry model for bilarge leptonic mixing and the lepton masses, Nucl. Phys. B 643 (2002) 247 hep-ph/0206087;

G. Seidl, Deconstruction and bilarge neutrino mixing, hep-ph/0301044. 